\title{
First in-vivo trials of a fiber Bragg grating based temperature profiling system
}

\author{
D. J. Webb \\ M. W. Hathaway \\ D. A. Jackson \\ Applied Optics Group, The University \\ Canterbury, Kent \\ CT2 7NR, United Kingdom
}

\section{S. Jones}

Cancer Research Institute, Perth, Australia

\section{Zhang}

I. Bennion

Photonics Research Group, Aston University

United Kingdom

\begin{abstract}
We describe the results of in-vivo trials of a portable fiber Bragg grating based temperature profile monitoring system. The probe incorporates five Bragg gratings along a single fiber and prevents the gratings from being strained. Illumination is provided by a superluminescent diode, and a miniature CCD based spectrometer is used for demultiplexing. The CCD signal is read into a portable computer through a small A/D interface; the computer then calculates the positions of the center wavelengths of the Bragg gratings, providing a resolution of $0.2^{\circ} \mathrm{C}$. Tests were carried out on rabbits undergoing hyperthermia treatment of the kidney and liver via inductive heating of metallic implants and comparison was made with a commercial Fluoroptic thermometry system. (o) 2000 Society of Photo-Optical Instrumentation Engineers. [S1083-3668(00)00701-2]
\end{abstract}

Keywords: Fiber Bragg grating; medical temperature sensor.

Paper JBO-90035 received June 14, 1999; revised manuscript received Sep. 21, 1999; accepted for publication Oct. 11, 1999.

\section{Introduction}

Recently, there has been an increased interest in thermal therapies and methods and a growing diversity in their applications in many specialist areas of medicine and dentistry. In many cases, techniques have been developed for ablating tissue using physical agents such as radio frequency (rf) currents, microwaves, lasers, and ultrasound to induce temperatures several tens of ${ }^{\circ} \mathrm{C}$ above the normal body level for periods of a few seconds to tens of seconds. In other cases, the required temperature increases are more modest and treatment temperatures of $40-50^{\circ} \mathrm{C}$ are required for periods of several minutes to an hour.

Sample applications include transcatheter rf ablation of cardiac tissue, ${ }^{1}$ rf ablation as a treatment for menorrhagia, a common problem comprising approximately $20 \%$ of outpatient referrals to gynaecological departments, ${ }^{2,3}$ treatment of vascular disease using the technique of thermally mediated balloon angioplasty, ${ }^{4,5}$ treatments of prostate disease. ${ }^{6,7}$ Furthermore, the addition of hyperthermal treatment to radiotherapy or chemotherapy in the treatment of breast cancer, melanoma, and some inoperable tumours of the rectum, bladder, and cervix has been shown to increase response rates significantly. ${ }^{8-10}$ There is also a need for thermometry in techniques involving laser- and microwave-induced heating in dental processing. ${ }^{11,12}$

A common feature of all thermal therapies and procedures is the need for temperature measurement to ensure safe and effective practices. ${ }^{13-20}$ In most cases, the provision of accurate thermometry is a demanding problem. In view of the invasive procedures involved, temperature probes should be as small as possible to minimize patient trauma and ideally should provide temperature information from many points along their length. Small sensors also have the advantage of short response times. In addition, measurements are often

Address correspondence to D. J. Webb; electronic mail: d.j.webb@ukc.ac.uk made in an environment of high electric field or ultrasonic pressure field in which temperature gradients of tens of ${ }^{\circ} \mathrm{C} / \mathrm{cm}$ exist. If artefacts are to be minimized, it is desirable that probes should be nonmetallic (to minimize interaction with electromagnetic fields and to reduce thermal conduction along the probe).

The use of thermocouples, thermistors, and optical thermometry systems during thermal treatments and procedures has been reported. There are many problems with conventional metallic sensors in the presence of electromagnetic fields, while optical thermometers commercially available for medical purposes are expensive and provide very few sensors. $^{21}$

For these reasons, we have been investigating the use of fiber Bragg gratings as medical temperature sensors. We have previously reported two signal processing systems that are able to recover the wavelength shifts associated with several gratings closely spaced along a single fiber. One uses interferometric wavelength shift detection ${ }^{22}$ for measuring the shift, while a miniature monochromator permits wavelength demultiplexing of the gratings; ${ }^{23}$ the other uses a scanned Fabry-Perot tuneable filter for both wavelength shift measurement and demultiplexing. ${ }^{24}$

More recently, in an effort to reduce the potential cost of a temperature profiling system, we have been investigating the use of an alternative scheme using a CCD-based spectrometer to carry out demultiplexing and to track the wavelength shift of the Bragg gratings. ${ }^{25,26}$ This paper reports on the construction of a portable system using this approach and describes the results of our first in-vivo trials, undertaken at the Cancer Research Institute in Perth, Australia.

\section{System Design}

The system is illustrated schematically in Figure 1. Light backreflected from the grating array is imaged by a spectrom-

1083-3668/2000/\$15.00 @ 2000 SPIE 


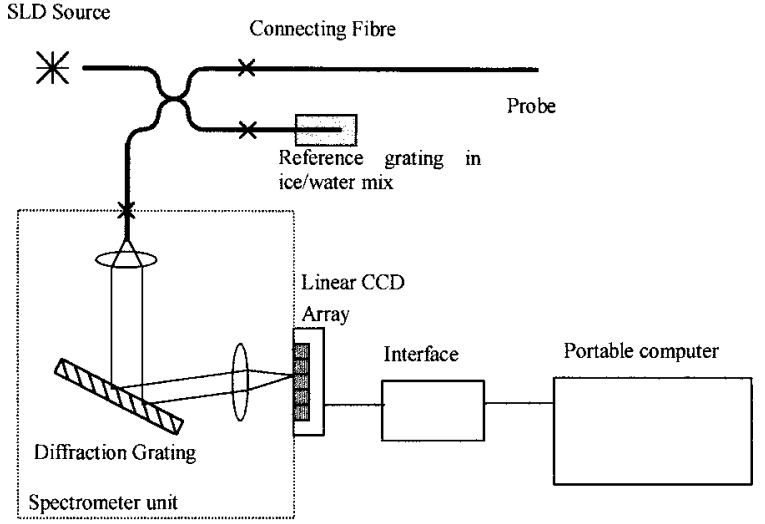

Fig. 1 System schematic. Crosses on fiber indicate the locations of demountable connectors.

eter onto a linear CCD array. In this way, the peaks associated with each grating are physically separated on the $\mathrm{CCD}$ and the change in the Bragg wavelength may be deduced by monitoring the movement of the peaks over the CCD. The difficulty with this approach is that while the grating spectra are typically $0.2 \mathrm{~nm}$ in width, a resolution of $0.1^{\circ} \mathrm{C}$ demands monitoring the shift in the mean wavelength to around $1 \mathrm{pm}$, usually corresponding to a small fraction of the pixel-pixel separation on the CCD array. Thus some forms of curve fitting or centroid algorithm must be used to determine the center wavelength with great precision, but even then the system is very susceptible to errors caused, for example, by mechanical creep or thermal expansion. To counter this, a reference grating at constant temperature was included in the system and all peak positions on the CCD array are measured from that one.

A miniature monochromator $(10 \times 15 \times 8 \mathrm{~cm})$ was designed and constructed. This used a folded path geometry to minimize size and weight and provided a dispersion of around $15 \mathrm{~nm}$ across a Thomsen CSF TH8711 1728 element linear CCD array, that could be bolted to its side; see Figure 2. A custom interface was constructed to link the raw CCD output to the parallel port of a laptop computer, the interface being controlled by a Visual BASIC program that read in the data from the CCD array and monitored the position of the spectral peaks corresponding to the Bragg gratings. To precisely lo-

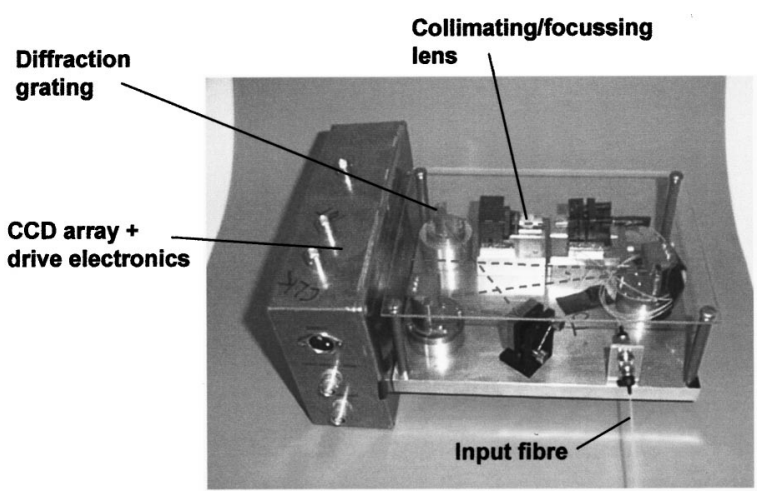

Fig. 2 Image of monochromator with CCD unit attached. The dashed line shows the free space optical path within the unit.

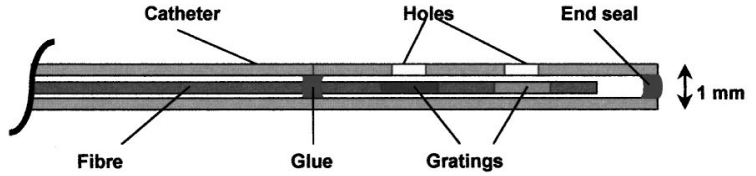

Fig. 3 Probe design. For clarity, only two grating elements are shown.

cate the peaks, the spectrum was divided up into a number of regions, each of which held just one grating. For each region, a fast Fourier transform of the sampled data was carried out yielding over 500 frequency components from which the first 15 were used to synthesize a low-pass-filtered version of the data. The peak of this synthesized function was obtained from the Fourier coefficients and used as the Bragg wavelength. This approach allowed the system to be used with a variety of peak shapes, resulting from nonuniform gratings as well as aberrations in the optical system.

The optical source was a $0.5 \mathrm{~mW}$ pigtailed superluminescent diode emitting around $830 \mathrm{~nm}$ and powered by an OEM diode power supply and temperature controller, which simply required connection to a $15 \mathrm{~V}$ laboratory PSU. The source was spliced to a coupler terminated with FC/PC connectors, which enabled easy assembly of the system and facilitated the attachment of different probes and reference gratings.

A key element of the system is the probe, which must prevent the fiber from being strained, protect against the possibility of the fiber being broken and left in the tissue and also permit good thermal contact with the surroundings. Our design for the probe is illustrated in Figure 3. The fiber was glued into a length of standard $1 \mathrm{~mm}$ o.d. surgical catheter, the end of which had already been prepared by having $\sim 1$ $\mathrm{mm}$ length holes cut into it to correspond with the grating positions (to facilitate good thermal contact). The fiber was approximately $5 \mathrm{~mm}$ shorter than the catheter length and the end of the catheter was closed with glue; this gave the fiber plenty of room to move within the catheter to relieve strain. The probe used for the majority of the trials had five gratings of $1 \mathrm{~mm}$ length on a $5 \mathrm{~mm}$ pitch. A sample spectrum read from the CCD is shown in Figure 4. The probe grating wavelengths as measured on an OSA at room temperature were $829.4,830.9,832.4,833.9$, and $835.4 \mathrm{~nm}$ and the reference grating was situated at $824.2 \mathrm{~nm}$.

\section{Laboratory Testing}

Basic testing took place in the laboratory prior to the in-vivo trials. A typical calibration run for one grating is illustrated in

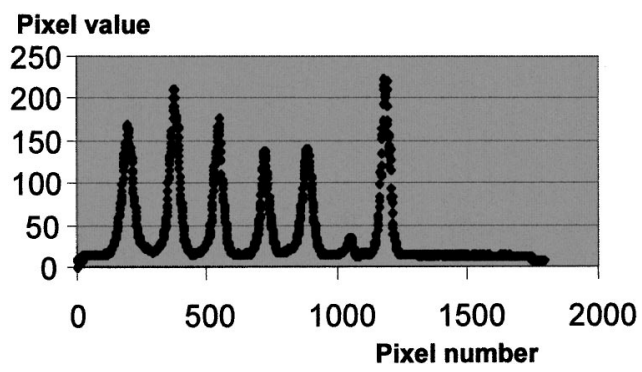

Fig. 4 Sample CCD trace showing five sensor gratings (on left) plus a reference grating (on right). 


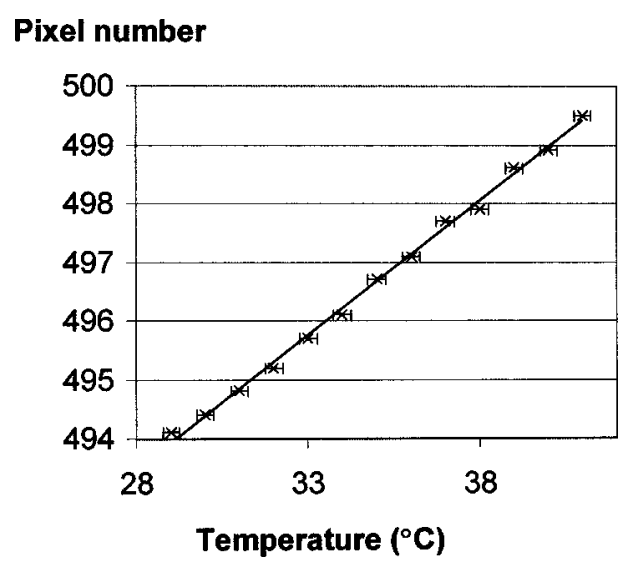

Fig. 5 Sample calibration run for one grating element. Error bars indicate uncertainty in the temperature of the water bath used. Also shown is the best linear fit through the points.

Figure 5, while an idea of the resolution can better be obtained from Figure 6, which shows fluctuations over a ten minute period, with an rms deviation about the mean value of $0.2^{\circ} \mathrm{C}$. In order to get the performance illustrated in Figures 5 and 6 , it was necessary to ensure that the signal level on the CCD was close to (but not at) saturation. This was because the DAC in the interface unit only possessed 8-bit resolution. To obtain good signals from both reference and probe gratings, it was necessary to carefully position appropriate neutral density filters in the spectrometer attenuating the reference by a different amount to the probe.

The system as designed was portable enough to be carried in a hold-all as hand luggage on the flight from London to Perth. On arrival, the unit was tested and found to be operating normally. However, while preparing for the actual trials, an unforeseen problem was noticed. Following switch-on, there was a steady long term drift in the pixel readout that only stabilized after about an hour (see Figure 7). This had not been seen during the initial testing in the UK as the unit was usually left switched on. Fortunately, the reading seemed to be stable after that time, but nonetheless during the trials, the

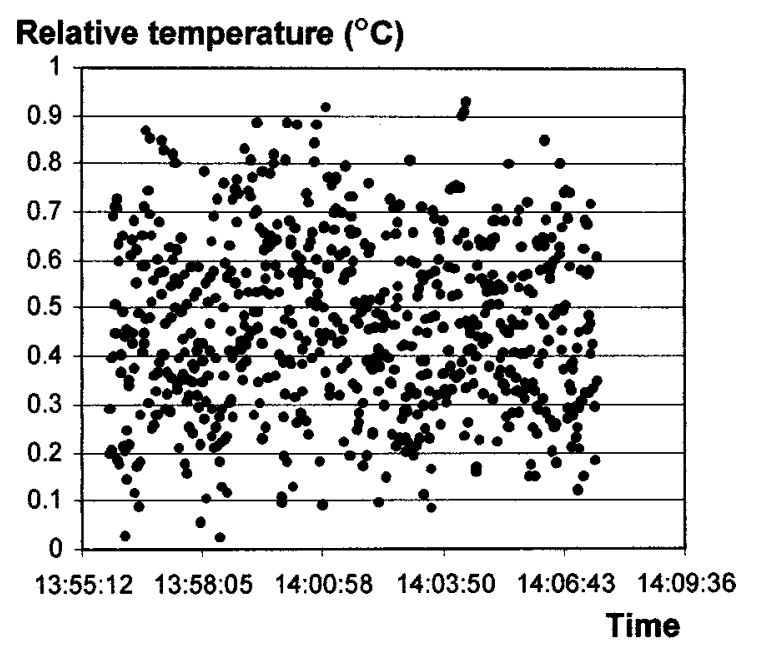

Fig. 6 Temperature fluctuations over a ten minute period.

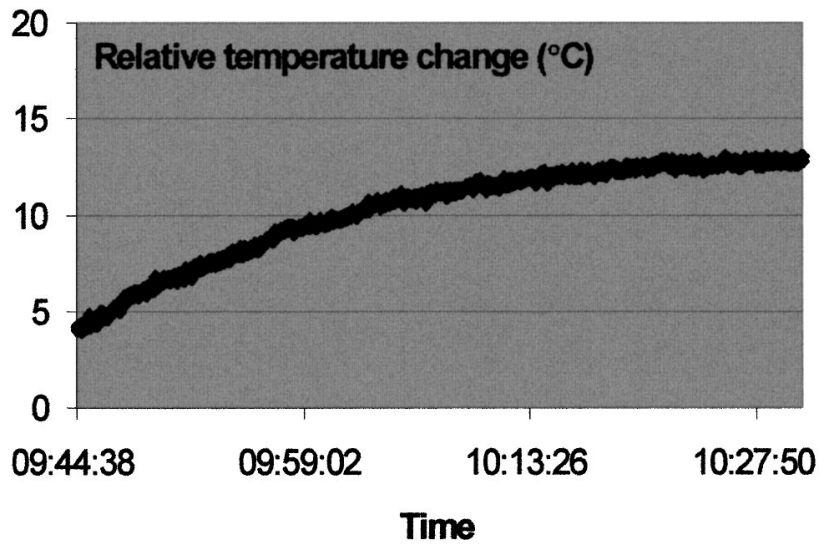

Fig. 7 Illustrating the reading drift following switch on from cold.

data were referenced to a pixel reading taken with the probe in ice water immediately following (or preceding) the data run.

Three weeks prior to the visit, several rabbits had been implanted with pieces of VX2 carcinoma in the liver so that reasonable sized tumours were available for some of the tests. Other tests were carried out on the healthy kidneys of these animals. The animals were initially anaesthetized and their organs exposed via a midline incision of the abdomen, enabling the inductive heating elements to be inserted in either the liver or kidney. Following the implantation of the temperature probe (often in conjunction with a reference probe from a Luxtron optical thermometer), the abdomen was sutured up to keep the organs at close to normal body temperature, prior to heating. In all cases, the animals were kept anaesthetised for the duration of the experiments and sacrificed immediately afterwards.

Part of the aim of the trial was to devise and test a suitable procedure for probe implantation; the following was found to be successful. The probe was initially inserted within a hypodermic needle and held in place with tape so that the probe end was several millimetres short of the needle end. The needle/probe was then inserted into the tissue and the needle withdrawn some distance along the fiber (out of the inductive field), leaving the probe in place. In practice, this procedure was quite straightforward. Following implantation, there was little resistance to the probe being moved in and out so it had to be held in place using tape. This lack of friction suggested that little axial stress would be communicated to the catheter, let alone the fiber inside. The implantation procedure is illustrated in Figure 8.

In the first trial, a major problem was encountered in that the field from the large alternating currents used in the inductive heating system was picked up by the sensing system resulting in a large noise, which all but smothered the peaks seen on the trace of Figure 4. We guessed that the most likely situation was that the field was being picked up by the interface electronics between the CCD unit, which in our system had to be close to the probe, and the computer. The problem was mainly solved by moving the interface and computer to the other side of the room and using long BNC leads to connect to the CCD array. In addition, software averaging over five scans was used to further reduce the noise to a level comparable with that in the benign laboratory environment 


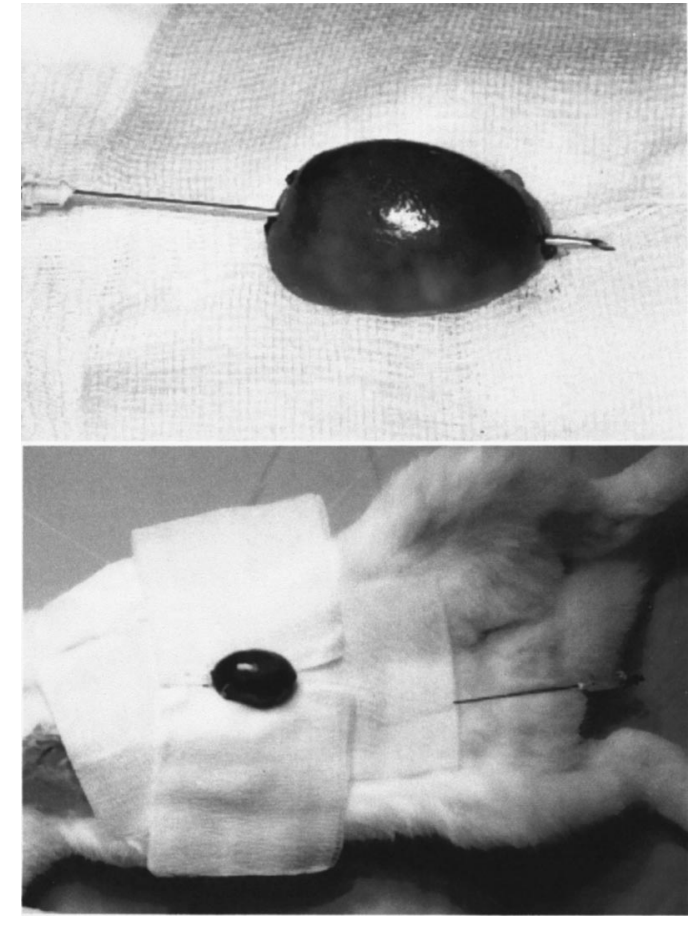

Fig. 8 Probe implantation procedure. Upper image: needle containing probe inserted in kidney, lower image: needle being retracted along fiber leaving probe in place.

while still permitting approximately a $1 \mathrm{~Hz}$ temperature acquisition rate.

Two other problems came to light during the course of the trials. The first was that several anomalous temperature readings were eventually attributed to the ice melting in the reference grating Dewar flask. This is not a serious problem, since in a fully engineered system, the reference grating might be held at constant temperature using a Peltier heat pump with a thermistor reference.

The second problem was more serious and indeed it was a surprise to us that we had not encountered it prior to the trials. Some - though not all—of the calibration runs turned out to be reproducibly nonlinear; see Figure 9, for example. We now attribute this to the following effect. There will be a number of features of the spectrum illustrated in Figure 3 which are constant in position. These include any ripple on the source

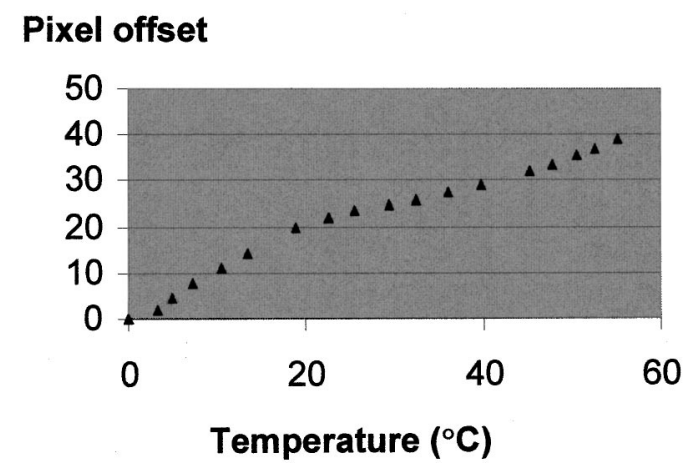

Fig. 9 Nonlinear grating calibration curve.

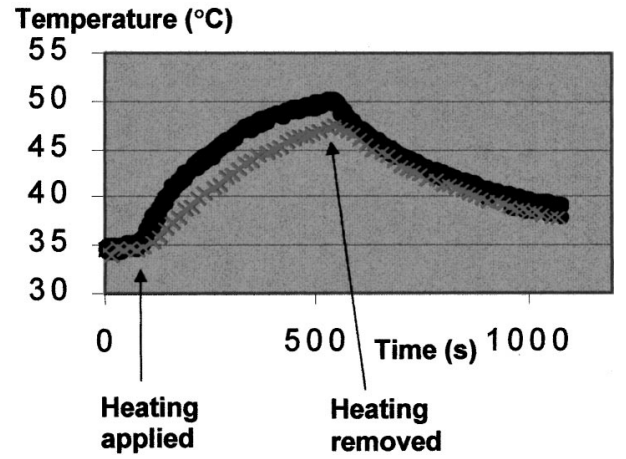

Fig. 10 Temperature recovered from FBG probe element (circles) collocated with Luxtron probe element (crosses).

spectrum, variations in the responsivity of the individual CCD pixels and attenuation which varies with position due, for example, to dust on the neutral density filers or mirrors. This means that as a grating peak moves across the CCD due to a temperature change, the detailed shape of that peak will change. We are measuring very small shifts in the peak position $(\sim 1 / 100$ of the width), so any variation in the shape to which we are fitting is likely to affect the value returned as the center of the peak.

We surmise this effect is also responsible for the long term drift in the reading shown in Figure 7. Here, the problem could be attributed to drift in the source spectrum due to heating following the switching on of the system. We are currently working on approaches to overcome these problems.

Several in-vivo experiments took place over a two-day period, which involved carrying out hyperthermia on both diseased livers and healthy kidneys. The FBG system reported temperature variations in line with those expected. Generally, it was not possible to draw quantitative conclusions concerning the accuracy of the data, as the background tissue temperature and heating varies with position in an unpredictable way. However, effort was made to locate a commercial Luxtron fluoroptic thermometer probe immediately adjacent to one of the FBG elements. The result of such a run is shown in Figure 10. The data show the increase in temperature following the application of inductive heating and the decrease that follows cessation of that heating. No attempt was made here to compensate for nonlinear calibration of the sort described in Figure 9, the FBG temperature was simply calculated from the mean gradient of its calibration curve along with the pixel reading corresponding to $0^{\circ} \mathrm{C}$. In view of this, we feel that the correspondence is very good. The general heating and cooling cycles are clearly illustrated and the maximum deviation of $3^{\circ} \mathrm{C}$ can be attributed to a combination of the nonlinear response along with the fact that we had no way of checking exactly how close together were the two probes.

There were some data from particular gratings where the recovered temperature showed a greater offset than this. We feel the reason here may be due to a buildup of dried tissue within the probe element, which could act to couple the fiber to the sleeving and hence restore strain sensitivity. This effect could be eliminated, either by having disposable probes, or by improving the probe construction to remove the slots. 


\section{Discussion}

We feel that the trial was very beneficial for a number of reasons:

(i) proof of the probe design being able to decouple the strain from the fiber;

(ii) development and proof of a suitable procedure for probe insertion;

(iii) proof that the system could function in the intense E.M. fields surrounding the hyperthermia unit;

(iv) proof of the ability to provide data comparable to the established Luxtron system;

(v) discovery of the non-linearities in the probe response described above.

We are currently working on means to improve the long term stability of the system and to remove or compensate for the nonlinear responses.

It is worth looking at two issues which are likely to affect the take up of this technology by the medical community: its comparative performance and its cost. The system is designed for applications where optical techniques offer the best (or only) solution, such as when intense electromagnetic fields are present; the case described in this paper is an obvious example. There are also many situations where thermometry must be carried out in a MRI machine. The Luxtron system mentioned earlier is probably currently the system of choice in the medical field, though it would also be possible to use interferometric techniques, such as an in-line fiber FabryPerot cavity. ${ }^{27}$

While it is clear that the scheme described in this paper still needs more engineering development before it is suitable for routine use, and therefore it is clearly at a disadvantage with regard to the Luxtron system, we believe we have demonstrated that such development is likely to be successful. The key feature of the use of Bragg gratings is that it allows multipoint measurement along a single optical fiber, which is important when issues of patient comfort are considered. This is not possible for the Luxtron technology. In principle, with some form of coherence multiplexing it would be possible to use a concatenated series of fiber Fabry-Perot interferometers with different cavity spacings to provide the same information, but the signal processing would be more complex and the practical construction of the sensor would be rather tricky and probably not well suited to mass production.

Concerning the potential cost of one of our systems, we should split the discussion to cover separately the signal processing unit and the probe. We believe that with a $1 \mathrm{~Hz}$ update rate it would be possible to use an edge emitting lightemitting diode (LED) source. Commercial CCD-based spectrometer units are available with specifications suitable for this application. We estimate the total component cost (excluding PC) for a single unit to be around $\$ 4000$. One should bear in mind that the cost could be reduced considerably in bulk.

The cost of a commercial probe is currently determined by the gratings themselves, however, there is every reason to hope that when the use of gratings really becomes widespread, the cost will fall dramatically. If the probe cost can be brought under $\$ 100$, then it is likely to become feasible to make the probes disposable which is considered advantageous clinically.

Currently, the hyperthermia system used in this work is being scaled up to permit human trials and it is intended that an improved version of the temperature sensor should be available for use with that system.

\section{Acknowledgments}

The authors would like to acknowledge support from the Wellcome Trust (043154/Z/94/ZIMP/RF/PK) for the development of this system. The trials were made possible through a travel grant from the UK Engineering and Physical Sciences Research Council.

\section{References}

1. K. H. Kuck and M. Akhtar, "New horizons for electrical therapy in managing ventricular and supraventricular tachyarrhythmias,' PacePacing Clin. Electrophysiol 16(3(II)), 503-505 (1993).

2. J. H. Phipps, B. V. Lewis, T. Roberts, M. V. Prior, J. W. Hand, M. Elder, and S. B. Field, "Treatment of functional menorrhagia by radiofrequency-induced thermal endometrial ablation,' Lancet $\mathbf{3 3 5}$, 374-376 (1990).

3. M. V. Prior, J. H. Phipps, T. Roberts, B. V. Lewis, J. W. Hand, and S. B. Field, "Treatment of menorrhagia by radiofrequency heating," Int. J. Hyperthermia 7(2), 213-220 (1990).

4. D. R. Fram, L. D. Gillam, T. A. Aretz, R. V. Tangco, J. F. Mitchel, J. P. Fisher, B. W. Sanzobrino, F. J. Kiernan, S. Nikulasson, A. Fieldman, and R. G. McCay, "Low pressure radiofrequency balloon angioplasty: evaluation in porcine peripheral arteries,' J. Am. Coll. Cardiol. 21(6), 1512-1521 (1993).

5. D. R. Fram, T. A. Artez, J. F. Mikan, A. Raisner, J. F. Mitchel, L. D. Gillam, D. D. Walters, and R. G. McCay, "In vivo radiofrequency balloon angioplasty of porcine coronary arteries: histologic effects and safety,', Am. Heart J. 126, 969-978 (1993).

6. M. L. Blute, K. M. Tomera, D. K. Hellerstein, C. E. McKiel, J. H. Lynch, J. B. Regan, and N. E. Sankey, "Transurethral thermotherapy for management of benign prostatic hyperplasia; results of the United States Prostaton Cetas (1990) cooperative study,' J. Urol. (Baltimore) 150, 1591-1596 (1993).

7. J. S. Rasor, A. R. Zlotta, S. D. Edwards, and C. C. Schulman, “Transurethral needle ablation (TUNA): thermal gradient mapping and comparison of lesion size in a tissue model and in patients with benign prostatic hyperplasia,' Eur Urol. 24(3), 411-414 (1993).

8. C. C. Vernon, C. van der Zee, and F.-F. Lui, 'Collaborative Phase III superficial hyperthermia trial,' in Proceedings of 14th Meeting of the European Society for Hyperthermic Oncology, Amsterdam, June 1994. Abstract Book p. 17 (1994).

9. J. Overgaard, D. Gonzalez, M. C. C. M. Hulshof, C. Arcangeli, O. Dahl, O. Mella, and S. M. Bentzen, "Randomized trial of hyperthermia as adjuvant to radiotherapy for recurrent or metastatic malignantmelanoma,' Lancet 345(8949), 540-543 (1995).

10. J. van der Zee and C. C. van Thoom, "Einverslag Ontwikkelingsgeneeskunde project OG89-23: de waarde van hyperthermic bij toevoeging aan radiotherapie bij de behandeling van inoperable en stralingresistent tumoren,' a report to the Dutch Health Ministry (1993).

11. P. F. Hogan and T. Mori, "Development of a method of continuous temperature measurement for microwave denture processing," Dent Mater. J. 9(1), 1-11 (1990).

12. J. Neev, A. Stabholtz, L. H. Liaw, M. Torabinejad, J. T. Fujishige, P. D. Ho, and M. W. Berns, "Scanning electron microscopy and thermal characteristics of dentin ablated by a short-pulse $\mathrm{XeCl}$ excimer lasers,', Lasers Surg. Med. 13(3), 353-362 (1993).

13. P. T. Finger, P. D. Smith, R. W. Paglione, and H. D. Perry, "Transscleral microwave cyclodestruction,', Invest. Ophthalmol. Visual Sci. 31(10), 2151-2155 (1990)

14. D. E. Haines, "The biophysics of radiofrequency catheter ablation in the heart: the importance of temperature monitoring,' Pace-Pacing Clin. Electrophysiol. 16(3 Pt 2), 586-591 (1993).

15. J. W. Hand, J. J. W. Lagendijk, J. B. Andersen, and J. C. Bolomey, 
"Quality assurance guidelines for ESHO protocols," Int. J. Hyperthermia 5(4), 421-428 (1989).

16. P. D. Inman and W. W. Brown III, "Uterine surface temperature changes caused by electrosurgical endometrial coagulation," J. Reprod. Med. 37(8), 667-670 (1992).

17. E. Kongsgaard, A. Foerster, H. Aass, and J. P. Amlie, "The effect of temperature-guided radiofrequency ablation of ventricular myocardium," Eur. Heart J. 14(6), 852-848 (1993).

18. J. J. Langberg, H. Calkins, R. el-Atassi, M. Borganelli, A. Leon, S. J. Kalbfleisch, and F. Morady, "Temperature monitoring during radiofrequency catheter ablation of accessory pathways," Circulation 86(5), 1469-1474 (1992)

19. G. Lee, H. S. Cohen, J. L. Rink, J. G. Roche, E. S. Hanna, and D. T. Mason, "Application of a new real-time temperature control pulsed laser for ablation of atherosclerotic plaque," Am. Heart J. 124(6), 1653-1654 (1992).

20. C. F. U. P. Wong, "The temperature for fusing blood vessels using a copper vapor laser,' Aust. Phys. Eng. Sci. Med. 14(2), 81-85 (1991).

21. T. C. Cetas, "Thermometry," in An Introduction to the Practical Aspects of Clinical Hyperthermia, S. B. Field and J. W. Hand, Eds., pp. 423-477, Taylor \& Francis, London (1990).
22. A. D. Kersey, T. A. Berkoff, and W. W. Morey, "Fiber optic Bragg grating strain sensor with drift-compensated high-resolution interferometric wavelength-shift detection,' Opt. Lett. 18, 72-74 (1993).

23. Y. J. Rao, D. J. Webb, D. A. Jackson, L. Zhang, and I. Bennion, "In-fiber Bragg-grating temperature sensor system for medical applications," J. Lightwave Technol. 15(5), 779-785 (1997).

24. Y. J. Rao, D. J. Webb, D. A. Jackson, L. Zhang, and I. Bennion, "Optical in-fiber Bragg grating sensor systems for medical applications," J. Biomed. Opt. 3(1), 38-44 (1998).

25. C. G. A. Askins, M. A. Putnam, and E. J. Friebele, "Instrumentation for interrogating many-element fiber Bragg grating arrays," Proc. Soc. Photo-Opt. Instrum. Eng. 2444, 257 (1995).

26. A. Ezbiri, S. E. Kanellopoulos, and V. A. Handerek, "High resolution instrumentation system for fiber-Bragg grating aerospace sensors," Opt. Commun. 150, 43-48 (1998).

27. P. Akhaven Leilabady and M. Corke, "All-fiber-optic remote sensing of temperature employing interferometric techniques," Opt. Lett. 12(10), 772-774 (1987). 